Check for updates

Cite this: Chem. Sci., 2019, 10, 9735

๑ All publication charges for this article have been paid for by the Royal Society of Chemistry

Received 15th April 2019

Accepted 23rd August 2019

DOI: $10.1039 / \mathrm{c} 9 \mathrm{sc} 01845 \mathrm{j}$

rsc.li/chemical-science

\section{A fluorinated alloy-type interfacial layer enabled by metal fluoride nanoparticle modification for stabilizing Li metal anodes $\uparrow$}

\author{
Feng Li,,$^{a}$ Yi-Hong Tan, $\dot{t}^{\mathrm{b}}$ Yi-Chen Yin, ${ }^{\mathrm{b}}$ Tian-Wen Zhang, ${ }^{a}$ Lei-Lei Lu, ${ }^{\mathrm{b}}$ \\ Yong-Hui Song, ${ }^{a}$ Te Tian, ${ }^{b}$ Bao Shen, ${ }^{\text {' }}$ Zheng-Xin Zhu ${ }^{b}$ and Hong-Bin Yao (D) *ab
}

\begin{abstract}
Using highly dispersed metal fluoride nanoparticles to construct a uniform fluorinated alloy type interfacial layer on the surface of Li metal anodes is realized by an ex situ solution chemical modification method. The fluorinated alloy-type interfacial layer can effectively inhibit the growth of undesirable Li dendrites while enhancing the performance of Li metal anodes.
\end{abstract}

Lithium metal batteries (LMBs) are considered as one of the most attractive candidates for next generation energy storage systems due to the high energy density provided by Li metal anodes..$^{1-3}$ However, the uncontrolled growth of Li dendrites on the surface of Li metal anodes would lead to piercing of the separator and cause a battery short-circuit, which severely hinders the practical applications of LMBs. ${ }^{4}$ The growth of $\mathrm{Li}$ dendrites mainly stems from an inhomogeneous and fragile interfacial layer formed via spontaneous reaction between $\mathrm{Li}$ metal and the electrolyte, which is named the solid electrolyte interphase (SEI). ${ }^{5}$ The SEI layer plays a vital role in inhibiting further reaction between the Li metal anode and the electrolyte due to its electron-insulating and ion-conducting properties. The fabrication of an electrochemically and mechanically stable SEI layer on the surface of Li metal anodes is presently considered as one of the most effective strategies to stabilize $\mathrm{Li}$ metal anodes. To fully functionalize the SEI layer on the surface of Li metal anodes, the SEI layer should also possess high elastic modulus, high thermodynamic stability, and a dense structure. ${ }^{6}$

In the past decade, much effort has been devoted to achieving desirable properties in SEI layers. Various electrolyte additives, ${ }^{7-9}$ all-fluorinated non-aqueous electrolytes ${ }^{10,11}$ and new-type lithium salts with a fluorine group ${ }^{12,13}$ have been introduced to reinforce the SEI layer and such a highly fluoridated protective layer was found to be highly effective for suppressing the growth of $\mathrm{Li}$ dendrites. It has also been demonstrated that the electrochemical performance and

${ }^{a}$ Division of Nanomaterials \& Chemistry, Hefei National Laboratory for Physical Sciences at the Microscale, University of Science and Technology of China, China

${ }^{b}$ Department of Applied Chemistry, CAS Center for Excellence in Nanoscience, Hefei Science Center of CAS, University of Science and Technology of China, 96 Jinzhai Road, Hefei, Anhui 230026, China. E-mail: yhb@ustc.edu.cn; Fax: +8655163607530 $\dagger$ Electronic supplementary information (ESI) available. See DOI: 10.1039/c9sc01845j

\$ F. L. and Y. H. T. contributed equally to this work. stability of LMBs can be improved impressively in the presence of inorganic fluorides (LiF) or organic fluorides (C-F). ${ }^{14}$ However, fluorinated additives and new-type salts used in the electrolytes are usually too expensive. Besides the in situ formation of advanced SEI layers by electrolyte modification, an ex situ fabrication of an artificial solid electrolyte interphase (ASEI) layer on the surface of Li metal anodes has been developed in the past few years. ASEI layers including hollow carbon spheres, ${ }^{15}$ two dimensional materials, ${ }^{16,17}$ functional polymers, ${ }^{18-20}$ nanocomposites, ${ }^{21,22}$ atomic layer deposition (ALD) layers ${ }^{23,24}$ and so on $^{7,25-29}$ were used to stabilize Li metal anodes. To some extent, these attempts have shown encouraging results to stabilize the surface of Li metal anodes during battery cycling. However, these ASEI layers cannot simultaneously possess high thermodynamic stability, good ionic conductivity, and capability of guiding Li metal deposition at one time to maximize their roles.

More recently, Li-based alloy type interfacial layers ${ }^{30}$ have been ex situ modified on the surface of Li metal anodes to guide uniform Li plating/stripping, leading to the improved cycling stability of Li metal anodes. The drawback of the alloy type layer is its limited electronic insulation to maintain the long term stability during repeated Li stripping/plating. In addition, as an important component in previously reported SEI layers, lithium fluoride (LiF) is highly electron-insulating and ultra-thermodynamically stable to reinforce the stability of SEI layers. ${ }^{6}$ It will be very interesting to combine $\mathrm{LiF}$ with Li-based alloy layers ${ }^{30-33}$ to further improve the performance of ASEI layers. However, the $e x$ situ modification of these combined ASEI layers is rarely reported due to the very low dissolubility of metal fluorides in the electrolyte. To make indissoluble materials highly dispersible in the solution, adopting the nanoparticle form is an efficient pathway. We also found that metal fluoride nanoparticles can be synthesized by a facile solution method ${ }^{34}$ on a large scale, implying that the cost of these nanoparticles is much lower than 
that of the fluorinated organics ${ }^{7-9}$ for the ex situ chemical modification of Li metal anodes.

Herein, we report the fabrication of a functional $\mathrm{LiF} / \mathrm{Li}-\mathrm{M}$ alloy layer on the surface of a Li metal anode via ex situ chemical modifications facilitated by highly dispersible metal fluoride nanoparticles $\left(\mathrm{MF}_{x} \mathrm{NPs}, \mathrm{M}=\mathrm{Zn}, \mathrm{Ca}, \mathrm{Mg}\right.$ or $\mathrm{Al}$ ). Based on the synergistic effect of the as-formed $\mathrm{LiF} / \mathrm{Li}-\mathrm{M}$ alloy composite layer, the protected $\mathrm{Li}$ metal anode exhibited an excellent cycling stability of 500 hours in symmetrical cells at a high current density of $1 \mathrm{~mA} \mathrm{~cm} \mathrm{~cm}^{-2}$ in conventional carbonate solvent based liquid electrolytes. Moreover, the LMB composed of the protected $\mathrm{Li}$ metal anode and $\mathrm{LiNi}_{0.8} \mathrm{Co}_{0.15} \mathrm{Al}_{0.05} \mathrm{O}_{2}$ (NCA) cathode displayed improved cycling stability $\left(147.5 \mathrm{~mA} \mathrm{~h} \mathrm{~g}^{-1}\right)$ in comparison to that of the pristine LMB $\left(20 \mathrm{~mA} \mathrm{~h} \mathrm{~g}^{-1}\right)$ at $0.5 \mathrm{C}$ after 150 cycles.

We carried out an ex situ chemical modification strategy to fabricate a functional $\mathrm{LiF} / \mathrm{Li}-\mathrm{M}$ alloy layer on the surface of the Li metal anode via immersing fresh $\mathrm{Li}$ metal foil into a suspension of $\mathrm{MF}_{x}$ NPs (Fig. 1a). The $\mathrm{MF}_{x}$ NPs were prepared by a facile solution method (for details please see the Experimental section $\dagger$ ). The transmission electron microscopy (TEM) images and X-ray diffraction (XRD) patterns show that the $\mathrm{MF}_{x}$ NPs synthesized by this facile solution method are uniform in size and pure phases (Fig. S1, ESI $\dagger$ ). Most importantly, the obtained $\mathrm{MF}_{x}$ NPs are highly dispersible in the co-solvent of 1,3dioxolane/1,2-dimethoxyethane (DOL/DME, $1: 1$, v/v) as shown in Fig. S2 (ESI $\dagger$ ), which can promote the reaction between $\mathrm{MF}_{x}$ NPs and fresh Li metal foil. The uniform ASEI protective layer consisting of $\mathrm{LiF} / \mathrm{Li}-\mathrm{M}$ alloy compounds can be built via the following reaction:

$$
\mathrm{Li}+\mathrm{MF}_{x} \rightarrow \mathrm{LiF}+\mathrm{Li}-\mathrm{M}(\mathrm{M}=\mathrm{Zn}, \mathrm{Ca}, \mathrm{Mg}, \mathrm{Al})
$$

As shown in Fig. 1b, a uniform black layer on the Li-metal surface can be obtained after being treated with the solution in which zinc fluoride nanoparticles $\left(\mathrm{ZnF}_{2} \mathrm{NPs}\right)$ were evenly dispersed. In contrast, there is no obvious change of fresh $\mathrm{Li}$
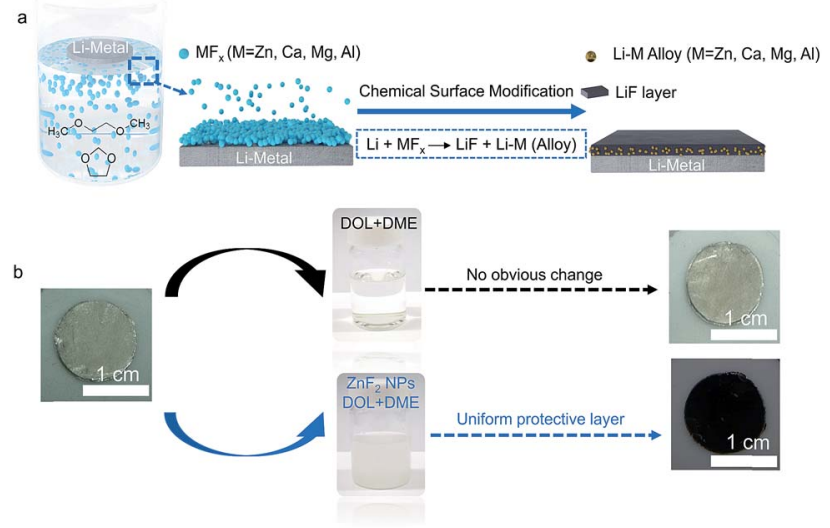

Fig. 1 (a) Schematic illustration of the ASEI layer formation via ex situ chemical modification. (b) Optical photographs of Li foil treated with co-solvent (DOL/DME) and co-solvent dispersed $\mathrm{ZnF}_{2} \mathrm{NPs}$, respectively. metal foil after being treated using pure DOL/DME co-solvent under the same conditions. The obvious distinction observed in Fig. 1b profoundly proves that the black protective layer is the product originating from the reaction between $\mathrm{ZnF}_{2}$ NPs and Li metal in DOL/DME solution. Furthermore, the optical photos of protected Li metal foil obtained by the treatment of other $\mathrm{MF}_{x}$ NPs $(\mathrm{M}=\mathrm{Ca}, \mathrm{Mg}, \mathrm{Al})$ in Fig. S3 (ESI $\dagger$ ) indicate the versatility of our proposed ex situ chemical modifications on the Li metal anode.

Scanning electron microscopy (SEM) was employed to analyze the surface morphological features of the Li metal anode after the treatment with $\mathrm{MF}_{x}$ NPs. The top-view and sideview SEM images (Fig. 2a-f and S4-S6, ESI $\dagger$ ) of Li metal foil treated with $\mathrm{MF}_{x}$ NPs both demonstrate that the uniform ASEI protective layers can be obtained by our developed nanoparticle treated strategy. In terms of Li metal foil treated with $\mathrm{ZnF}_{2} \mathrm{NPs}$, the ASEI protective layer is compact as shown in the top-view SEM images (Fig. 2a and b). The designed compact layer can effectively prevent the Li metal anode contact with the electrolyte, thereby suppressing uncontrolled side reactions and the consumption of the electrolyte. Besides, the thickness of the asfabricated ASEI protective layer was measured to be $5-10 \mu \mathrm{m}$ as shown in the side-view SEM images (Fig. $2 \mathrm{~d}$ and e), in which the $\mathrm{LiF} / \mathrm{Li}-\mathrm{Zn}$ alloy layer on the top is marked by cyan colour and the lithium under the alloy layer is marked by green colour. The obtained structure is similar to that of other reports related to the ASEI protective layer. ${ }^{35}$
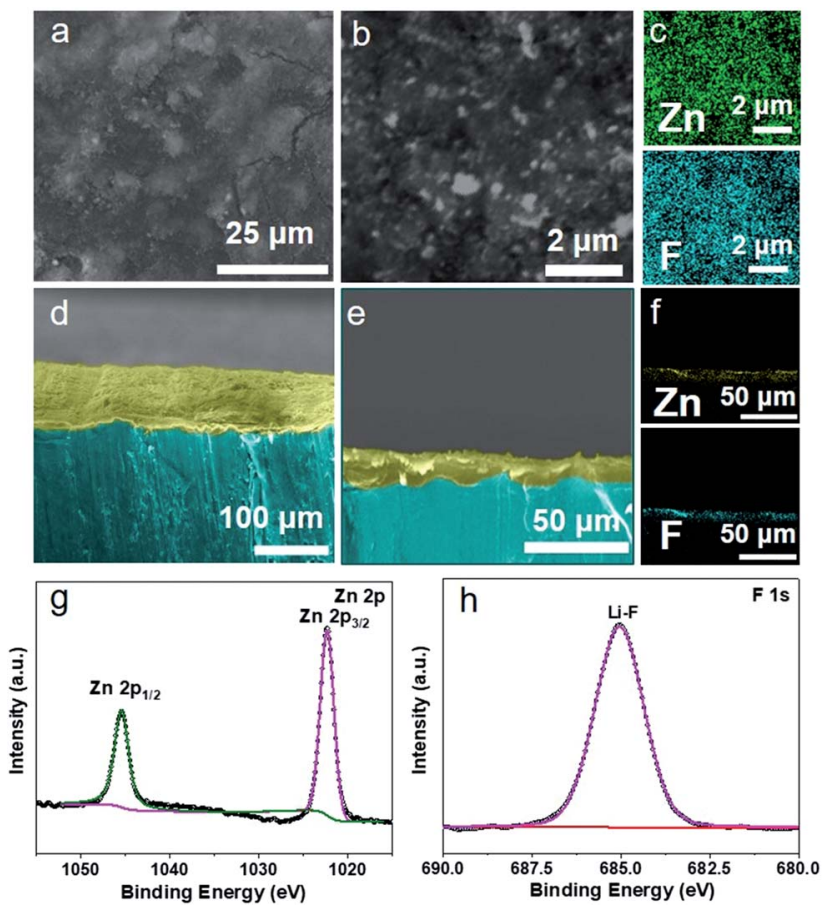

Fig. 2 (a and b) The top-view SEM images, (c) corresponding EDX mapping analysis, ( $d$ and e) side-view SEM images and ( $f$ ) corresponding EDX mapping analysis of $\mathrm{ZnF}_{2} \mathrm{NPs}$ - treated Li metal anodes, respectively. The modified layer is denoted by cyan colour and the $\mathrm{Li}$ is denoted by green colour. ( $g$ and $h$ ) The $Z n 2 p, F$ 1s XPS analysis of the corresponding ASEl layer. 
The components of the as-fabricated ASEI protective layer were further revealed by energy dispersive X-ray spectroscopy (EDX) mapping. As shown in Fig. 2c and f, F and $\mathrm{Zn}$ are homogeneously dispersed in the ASEI protective layer indicating that a uniform coating of the $\mathrm{LiF} / \mathrm{Li}-\mathrm{Zn}$ alloy layer was formed on the surface of the Li metal anode. Moreover, X-ray photoelectron spectroscopy (XPS) was used to explore the compositions and chemical bonding information in the ASEI protective layer (Fig. $2 \mathrm{~g}$ and h). As shown in Fig. $2 \mathrm{~g}$, the XPS spectrum of the $\mathrm{Zn} 2 \mathrm{p}$ orbital splits into two peaks at 1022.3 and $1045.4 \mathrm{eV}$, which belong to $\mathrm{Zn} 2 \mathrm{p}_{3 / 2}$ and $\mathrm{Zn} 2 \mathrm{p}_{1 / 2}$, respectively, corresponding to the $\mathrm{Zn}$ in the $\mathrm{Li}-\mathrm{Zn}$ alloy. ${ }^{36}$ Besides, the XPS signal of the F 1s split can be fitted to the peak located at 685.0 $\mathrm{eV}$, which is attributed to the formation of LiF. ${ }^{32}$ The results of EDX mapping and XPS can validly indicate that the as-formed ASEI protective layer is composed of the products of the reaction between the $\mathrm{ZnF}_{2}$ NPs and Li metal. Other $\mathrm{MF}_{x}$ NPs also show similar results on the surface of Li metal anodes to the $\mathrm{ZnF}_{2}$ NPs, and the analytical data such as EDX and XPS are shown in Fig. S4-S6 (ESI $\dagger$ ), respectively. All these results demonstrate that the high reactivity and good dispersability of $\mathrm{MF}_{x} \mathrm{NPs}(\mathrm{M}=\mathrm{Zn}$, $\mathrm{Ca}, \mathrm{Mg}, \mathrm{Al}$ ) can be used to realize the construction of the ASEI protective layer via an efficient solution chemical reaction with active Li metal.

The effectiveness of the as-formed ASEI protective layer for promoting the cycling stability of the Li metal anode was first revealed by the polarization voltage evolution behavior in symmetric cells. Fig. $3 \mathrm{a}$ and $\mathrm{b}$ show the huge difference in the behavior of polarization voltage variation as a function of time at a relatively high current density $\left(1 \mathrm{~mA} \mathrm{~cm}^{-2}\right)$ between the pristine Li metal anode and protected Li metal anode by the LiF/
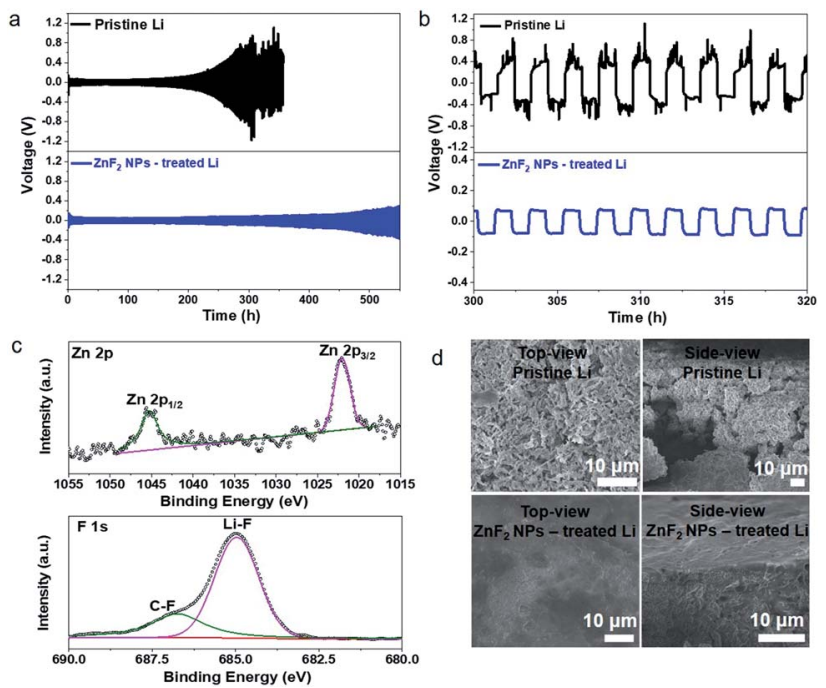

Fig. 3 (a) The evolution of voltage profiles of symmetric cells based on the pristine Li metal anode (top) and $\mathrm{ZnF}_{2} \mathrm{NPs}$ - treated Li metal anodes (bottom), respectively. (b) The corresponding voltage profiles of (a) in an enlarged view of 300-320 h. (c) The Zn 2p, F 1s XPS spectra of the ASEl layer formed on Li foil after the 10th cycle. (d) The surface and side-view SEM images of the pristine Li metal anode and $\mathrm{ZnF}_{2} \mathrm{NPs}$ - treated Li metal anodes after $320 \mathrm{~h}$ cycling.
Li-Zn alloy layer. The pristine Li metal anode exhibits a high polarization voltage $(\sim 200 \mathrm{mV})$ within $150-220 \mathrm{~h}$ and gradually increases to $400 \mathrm{mV}$ after $300 \mathrm{~h}$ cycling, indicating that the migration of lithium ions is severely impeded by the interface failure (top of Fig. 3a). In contrast, the Li metal anodes treated with $\mathrm{ZnF}_{2}$ NPs display long and stable voltage curves $(\sim 50 \mathrm{mV}$ within initial $220 \mathrm{~h}$ cycling) and can be cycled up to $400 \mathrm{~h}$ with a polarization voltage less than $100 \mathrm{mV}$ (bottom of Fig. 3a). In terms of cycle performances within $300-320 \mathrm{~h}$ as shown in Fig. $3 \mathrm{~b}$, the polarization voltage increases sharply and becomes unstable in the pristine $\mathrm{Li}$ metal anode, indicating the rapid degradation of the interface, whereas the protected Li metal still exhibits an extremely stable voltage profile with a low polarization voltage of $\sim 70 \mathrm{mV}$. Besides, other $\mathrm{MF}_{x} \mathrm{NP}$ modifications also have a similar function of stabilizing deposition behavior on the surface of Li metal anodes to the $\mathrm{ZnF}_{2}$ NPs (Fig. S7, ESI $†$ ). In our study system, the activation process may be related to the surface side reactions of the as-formed protective layer and the electrolyte. After the side reaction, a relatively stable solid electrolyte interphase (SEI) layer formed on the Li metal anode, leading to the stable plating/stripping cycling with a relatively lower overpotential. With the prolongation of cycling, the ASEI layer on the surface of Li metal was gradually damaged due to the repeated volume change of the Li metal anode and the overpotential increased. Thus, it seems that the polarization of the Li metal anode decreased to a lower value in the middle of the test. The obvious difference of plating/stripping voltage evolution of the pristine Li metal anode and protected Li metal anode during the cycling implies that the Li plating/stripping efficiency is promoted by the $\mathrm{LiF} / \mathrm{Li}-\mathrm{M}$ alloy layer, which can be attributed to the improved lithium ion surface transport by LiF $^{30}$ and preferred $\mathrm{Li}$ nucleation sites in the $\mathrm{Li}-\mathrm{M}$ alloy layer. Our developed LiF/alloy ASEI protective layer displayed the best efficiency to stabilize the Li metal anode among the recent reports using conventional carbonate solvent based liquid electrolytes (details in Table S1, ESI $\dagger$ ). The reduced polarization voltage of our ASEI layer protected lithium metal anode is also superior to the previous reports of solely LiF reinforced SEI layer protected Li metal anodes and comparable to the alloy layer protected Li metal anodes. ${ }^{31,37,38}$

In order to further verify the stability of the as-fabricated ASEI protective layer during the cycling, XPS was used to characterize the chemical information of the ASEI protective layer after the first ten cycles. As shown in Fig. 3c, there is no obvious variation in the peak positions and intensities of $\mathrm{Zn}$ and $\mathrm{F}$. The emergence of a small C-F peak can be observed due to the initial decomposition of the electrolyte during the activation process. ${ }^{32,39}$ The XPS results similar to those of the electrode before cycling (Fig. $2 \mathrm{~g}$ and $\mathrm{h}$ ) indicate that the $\mathrm{LiF} / \mathrm{Li}-\mathrm{Zn}$ alloy layer is stable during the $\mathrm{Li}$ plating/stripping processes. This also means that the $\mathrm{LiF} / \mathrm{Li}-\mathrm{Zn}$ alloy layer can not only effectively suppress the growth of Li dendrites but also prevent the contact between the underlying Li metal and the electrolyte to form extra thick and fragile SEI layers.

The surface morphology characterization by SEM shows that significant Li dendritic growth occurred on the surface of the pristine Li metal anode (Fig. 3d and S8, ESI $\dagger$ ). In contrast, the 
protected $\mathrm{Li}$ metal anode by the $\mathrm{LiF} / \mathrm{Li}-\mathrm{M}(\mathrm{M}=\mathrm{Zn}, \mathrm{Al})$ alloy layer after 160 and $320 \mathrm{~h}$ cycling still maintains a relatively smooth surface without dendrites (Fig. 3d, S9 and S10, ESI $\dagger$ ). Based on the SEM images and EDX mapping results of the Li metal anode at the different cycling times (Fig. S11, ESI $\dagger$ ), we can conclude that the protective layer can be maintained on the surface of the Li metal anode after short or long term cycling. This again indicates the stability and effectivity of our designed ASEI protective layer. In addition, the interfacial resistance of both the protected Li metal anode by the ASEI layer and the pristine Li metal anode exhibits a relatively small impedance before cycling (Fig. S12, ESI $\dagger$ ), confirming that the introduction of the ASEI protective layer does not deteriorate charge transfer at the interface. But the interfacial impedance of the pristine Li metal anode increases to $284 \Omega$ after $320 \mathrm{~h}$ cycling, which corresponds to a rapid increase in the polarization voltage (Fig. 3a and b), indicating the high diffusion resistance of $\mathrm{Li}^{+}$ions at the interface due to the rough surface and thickened SEI layer. In contrast, the protected Li metal anodes show no obvious change in the impedance before and after cycling, which is consistent with the smooth surface of protected $\mathrm{Li}$ metal anodes after cycling. The rapid ion diffusion and stable interfaces cause the low diffusion impedance of $\mathrm{Li}^{+}$ions under the effect of the ASEI protective layer during the plating/stripping process. Therefore, the $\mathrm{LiF} / \mathrm{Li}-\mathrm{M}$ alloy layer can effectively protect the $\mathrm{Li}$ metal anode providing the long-term cycling stability of the symmetric cells.

The full Li-metal-based cells (NCA/Li) were assembled and tested to demonstrate the effectiveness of the $\mathrm{LiF} / \mathrm{Li}-\mathrm{M}$ alloy layer in LMBs. As shown in Fig. 4a, the initial discharge specific capacities of all cells are higher than $200 \mathrm{~mA} \mathrm{~h} \mathrm{~g}{ }^{-1}$. The difference between initial discharge and charge capacity of the NCA cathode is caused by the uncontrolled side reactions between the electrode and electrolyte. ${ }^{40}$ In the 150 -cycle test at
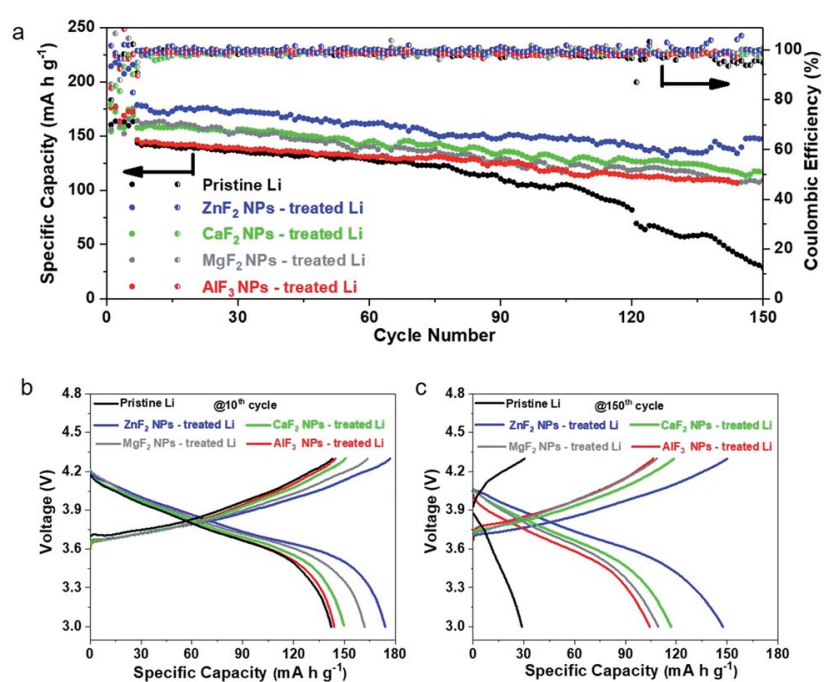

Fig. 4 (a) Electrochemical cycling performance at $0.5 \mathrm{C}$ and (b and $\mathrm{c}$ ) representative charge/discharge profiles of cells based on the pristine $\mathrm{Li}$ metal anode and MF $\mathrm{NPs}$ - treated Li metal anodes in the $10^{\text {th }}$ and $150^{\text {th }}$ cycle, respectively.
$0.5 \mathrm{C}\left(1 \mathrm{C}=200 \mathrm{~mA} \mathrm{~g}^{-1}, 1 \mathrm{C}\right.$ means a current density at which the theoretical specific capacity of the electrode material can be achieved in one hour), there is a significant performance difference between the cells based on the pristine Li metal anode and protected $\mathrm{Li}$ metal anode. The cell of all $\mathrm{LiF} / \mathrm{L}-\mathrm{M}$ alloy layers protected $\mathrm{Li}$ metal anodes can maintain stable discharge capacities with very slow capacity decay. By contrast, the discharge capacity of the cell using the pristine Li metal anode shows rapid decrease of capacity, which drops to nearly $20 \mathrm{~mA} \mathrm{~h} \mathrm{~g}^{-1}$ in the $150^{\text {th }}$ cycle. Detailed comparison of charge/ discharge voltage profiles further indicates that the polarization of the full cell based on the protected Li metal anode is lower than that of the cell using the pristine Li metal anode (Fig. $4 \mathrm{~b}$ and c). In particular, after the $150^{\text {th }}$ cycle, the polarization of the cell using the protected $\mathrm{Li}$ metal anode did not increase significantly but that of the cell using the pristine Li metal anode increased very much. The significantly improved cycling stability of the cell using the protected $\mathrm{Li}$ metal anode verified the effectiveness of the as-formed ASEI protective layers in practical LMBs.

In summary, we have proposed a $\mathrm{MF}_{x} \mathrm{NP}$ induced functional modification strategy to fabricate a $\mathrm{LiF} / \mathrm{Li}-\mathrm{M}(\mathrm{M}=\mathrm{Zn}, \mathrm{Ca}, \mathrm{Mg}$, $\mathrm{Al}$ ) alloy ASEI layer for stabilizing Li metal anodes. Due to the high activity of $\mathrm{MF}_{x}$ NPs, the $\mathrm{LiF} / \mathrm{Li}-\mathrm{M}$ alloy layer can be simultaneously formed on the surface of the Li metal anode by the reaction between $\mathrm{MF}_{x}$ NPs and Li metal. The ASEI protective layer can effectively inhibit the growth of Li dendrites at a high current density of $1 \mathrm{~mA} \mathrm{~cm}^{-2}$, realizing the long lifespan of 150 cycles in NCA matched LMBs. Our developed $\mathrm{MF}_{x}$ NP induced $\mathrm{LiF} / \mathrm{Li}-\mathrm{M}$ alloy ASEI layer will open a new avenue to construct artificial protective layers on Li metal anodes via the chemical modification of rationally designed nanoparticles.

\section{Conflicts of interest}

There are no conflicts to declare.

\section{Acknowledgements}

The authors acknowledge support from the National Natural Science Foundation of China (No. 51571184 and 21875236), the Fundamental Research Funds for the Central Universities (Grant WK2060190085), and the joint Funds from Hefei National Synchrotron Radiation Laboratory (Grant KY2060000111). This work was partially carried out at the USTC Center for Micro and Nanoscale Research and Fabrication, and we thank Xiu-Xia Wang and Hai-Tao Liu for their help in SEM and EDX analysis. H. B. Y. acknowledges the support from "the Recruitment Program of Thousand Youth Talents".

\section{Notes and references}

1 W. Xu, J. Wang, F. Ding, X. Chen, E. Nasybulin, Y. Zhang and J.-G. Zhang, Energy Environ. Sci., 2014, 7, 513-537.

2 L. Xu, S. Tang, Y. Cheng, K. Wang, J. Liang, C. Liu, Y.-C. Cao, F. Wei and L. Mai, Joule, 2018, 2, 1991-2015. 
3 S. Jiang, Y. Lu, Y. Lu, M. Han, H. Li, Z. Tao, Z. Niu and J. Chen, Chem.-Asian J., 2018, 13, 1379-1385.

4 X.-B. Cheng, R. Zhang, C.-Z. Zhao and Q. Zhang, Chem. Rev., 2017, 117, 10403-10473.

5 E. Peled, J. Electrochem. Soc., 1979, 126, 2047-2051.

6 X. B. Cheng, R. Zhang, C. Z. Zhao, F. Wei, J. G. Zhang and Q. Zhang, Adv. Sci., 2016, 3, 1500213.

7 C. Yan, Y. X. Yao, X. Chen, X. B. Cheng, X. Q. Zhang, J. Q. Huang and Q. Zhang, Angew. Chem., Int. Ed., 2018, 130, 14251-14255.

8 E. Markevich, G. Salitra, F. Chesneau, M. Schmidt and D. Aurbach, ACS Energy Lett., 2017, 2, 1321-1326.

9 X. Q. Zhang, X. B. Cheng, X. Chen, C. Yan and Q. Zhang, Adv. Funct. Mater., 2017, 27, 1605989.

10 X. Fan, L. Chen, O. Borodin, X. Ji, J. Chen, S. Hou, T. Deng, J. Zheng, C. Yang and S.-C. Liou, Nat. Nanotechnol., 2018, 13, 715.

11 L. Suo, W. Xue, M. Gobet, S. G. Greenbaum, C. Wang, Y. Chen, W. Yang, Y. Li and J. Li, Proc. Natl. Acad. Sci. U. S. A., 2018, 115, 1156-1161.

12 Q. Ma, Z. Fang, P. Liu, J. Ma, X. Qi, W. Feng, J. Nie, Y. S. Hu, H. Li and X. Huang, ChemElectroChem, 2016, 3, 531-536.

13 L. Li, S. Zhou, H. Han, H. Li, J. Nie, M. Armand, Z. Zhou and X. Huang, J. Electrochem. Soc., 2011, 158, A74-A82.

14 C. Wang, Y. S. Meng and K. Xu, J. Electrochem. Soc., 2019, 166, A5184-A5186.

15 G. Zheng, S. W. Lee, Z. Liang, H.-W. Lee, K. Yan, H. Yao, H. Wang, W. Li, S. Chu and Y. Cui, Nat. Nanotechnol., 2014, 9, 618.

16 Q. Zhang, Y. Wang, Z. W. Seh, Z. Fu, R. Zhang and Y. Cui, Nano Lett., 2015, 15, 3780-3786.

17 K. Yan, H.-W. Lee, T. Gao, G. Zheng, H. Yao, H. Wang, Z. Lu, Y. Zhou, Z. Liang and Z. Liu, Nano Lett., 2014, 14, 6016-6022.

18 K. Liu, B. Kong, W. Liu, Y. Sun, M.-S. Song, J. Chen, Y. Liu, D. Lin, A. Pei and Y. Cui, Joule, 2018, 2, 1857-1865.

19 R. Bouchet, S. Maria, R. Meziane, A. Aboulaich, L. Lienafa, J.-P. Bonnet, T. N. Phan, D. Bertin, D. Gigmes and D. Devaux, Nat. Mater., 2013, 12, 452.

20 Z. Liang, G. Zheng, C. Liu, N. Liu, W. Li, K. Yan, H. Yao, P.-C. Hsu, S. Chu and Y. Cui, Nano Lett., 2015, 15, 2910-2916.
21 F. Badway, F. Cosandey, N. Pereira and G. Amatucci, J. Electrochem. Soc., 2003, 150, A1318-A1327.

22 Q. Li, P. Xu, W. Gao, S. Ma, G. Zhang, R. Cao, J. Cho, H. L. Wang and G. Wu, Adv. Mater., 2014, 26, 1378-1386.

23 Y. Cao, X. Meng and J. W. Elam, ChemElectroChem, 2016, 3, 858-863.

24 C. F. Lin, A. C. Kozen, M. Noked, C. Liu and G. W. Rubloff, Adv. Mater. Interfaces, 2016, 3, 1600426.

25 C.-P. Yang, Y.-X. Yin, S.-F. Zhang, N.-W. Li and Y.-G. Guo, Nat. Commun., 2015, 6, 8058.

26 N. W. Li, Y. X. Yin, C. P. Yang and Y. G. Guo, Adv. Mater., 2016, 28, 1853-1858.

27 C. Yan, X. B. Cheng, Y. Tian, X. Chen, X. Q. Zhang, W. J. Li, J. Q. Huang and Q. Zhang, Adv. Mater., 2018, 30, 1707629.

28 C. Yang, Y. Yao, S. He, H. Xie, E. Hitz and L. Hu, Adv. Mater., 2017, 29, 1702714.

29 C. Yang, K. Fu, Y. Zhang, E. Hitz and L. Hu, Adv. Mater., 2017, 29, 1701169.

30 X. Liang, Q. Pang, I. R. Kochetkov, M. S. Sempere, H. Huang, X. Sun and L. F. Nazar, Nat. Energy, 2017, 2, 17119.

31 Z. Tu, S. Choudhury, M. J. Zachman, S. Wei, K. Zhang, L. F. Kourkoutis and L. A. Archer, Nat. Energy, 2018, 3, 310.

32 C. Yan, X. B. Cheng, Y. X. Yao, X. Shen, B. Q. Li, W. J. Li, R. Zhang, J. Q. Huang, H. Li and Q. Zhang, Adv. Mater., 2018, 30, 1804461.

33 Q. Pang, X. Liang, I. R. Kochetkov, P. Hartmann and L. F. Nazar, Angew. Chem., Int. Ed., 2018, 57, 9795-9798.

34 C. Feldmann, M. Roming and K. Trampert, Small, 2006, 2, 1248-1250.

35 K. Liao, S. Wu, X. Mu, Q. Lu, M. Han, P. He, Z. Shao and H. Zhou, Adv. Mater., 2018, 30, 1705711.

36 B. V. Christ, The Elements and Native Oxides, 2000.

37 R. Xu, X. Q. Zhang, X. B. Cheng, H. J. Peng, C. Z. Zhao, C. Yan and J. Q. Huang, Adv. Funct. Mater., 2018, 28, 1705838.

38 L. Fan, H. L. Zhuang, L. Gao, Y. Lu and L. A. Archer, J. Mater. Chem. A, 2017, 5, 3483-3492.

39 V. Shutthanandan, M. Nandasiri, J. Zheng, M. H. Engelhard, W. Xu, S. Thevuthasan and V. Murugesan, J. Electron Spectrosc. Relat. Phenom., 2019, 231, 2-10.

40 W. Luo, A. Calas, C. Tang, F. Li, L. Zhou and L. Mai, ACS Appl. Mater. Interfaces, 2016, 8, 35219-35226. 